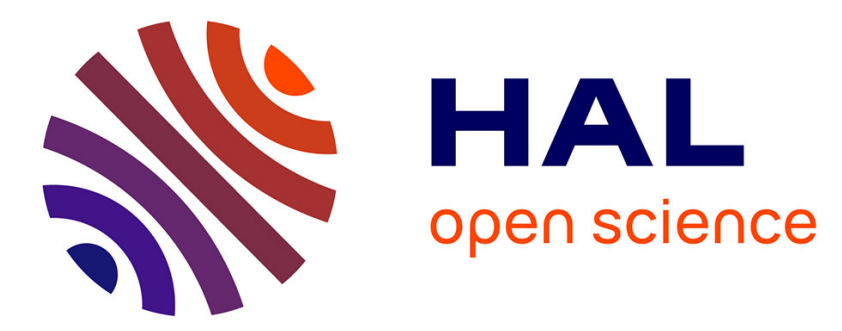

\title{
Carrier lifetime measurements from transient electrical photoresponses
}

\author{
D.M. Bielle-Daspet, A.M. Johan, F. Espioussas
}

\section{To cite this version:}

D.M. Bielle-Daspet, A.M. Johan, F. Espioussas. Carrier lifetime measurements from transient electrical photoresponses. Revue de Physique Appliquée, 1980, 15 (2), pp.219-227. 10.1051/rphysap:01980001502021900 . jpa-00244718

\section{HAL Id: jpa-00244718 https://hal.science/jpa-00244718}

Submitted on 1 Jan 1980

HAL is a multi-disciplinary open access archive for the deposit and dissemination of scientific research documents, whether they are published or not. The documents may come from teaching and research institutions in France or abroad, or from public or private research centers.
L'archive ouverte pluridisciplinaire HAL, est destinée au dépôt et à la diffusion de documents scientifiques de niveau recherche, publiés ou non, émanant des établissements d'enseignement et de recherche français ou étrangers, des laboratoires publics ou privés. 


\title{
Carrier lifetime measurements from transient electrical photoresponses
}

\author{
D. M. Bielle-Daspet, A. M. Johan and F. Espioussas \\ Centre d'Etude Spatiale des Rayonnements, B.P. 4346, 31029 Toulouse Cedex, France
}

\begin{abstract}
Résumé. - Les durées de vie mesurées à partir des photoréponses de conductivité (sous courant ou tension constants de polarisation), de courant de diffusion et de tension de circuit ouvert, créées par excitation transitoire de porteurs, sont considérées. Partant des expressions analytiques, la discussion porte sur les expressions clef, les conditions de validité et les applications de chacune des méthodes discutées, en supposant une excitation uniforme de porteurs dans un échantillon unidimensionnel et homogène; la discussion tient compte des caractéristiques de l'échantillon (épaisseur $W$, durée de vie volumique $\tau_{v}$, vitesses de recombinaison aux surfaces $s_{1,2}$, coefficient de diffusion $D$ des porteurs minoritaires et densité d'équilibre en porteurs majoritaires $p_{0}$ ), de la densité de porteurs en excès $\Delta p$, de la durée $T$ de l'impulsion lumineuse et des temps $t$ auxquels la réponse est considérée. L'étude expérimentale porte surtout sur des échantillons de silicium d'épaisseur jusqu'à $1 \mathrm{~mm}$, éclairés par des impulsions laser de durée $T=2$ à $30 \mathrm{~ns}$. Les réponses à une lumière pénétrante $\left(\lambda=1,06 \mu \mathrm{m}, \alpha=40 \mathrm{~cm}^{-1}\right.$ dans le silicium usuel; $\Delta p=10^{-4}$ à $\left.10^{2} p_{0}\right)$ et à une lumière fortement absorbée $\left(\lambda=0,69\right.$ ou $0,53 \mu \mathrm{m}, \alpha=2 \times 10^{3}$ et $10^{4} \mathrm{~cm}^{-1}$; intensité du faisceau $E_{0}=10^{-4}$ à $10^{3} \mathrm{~mJ} / \mathrm{cm}^{2}$ ) sont utilisées pour montrer sous quelles conditions les photoréponses observées conduisent aux mêmes valeurs mesurées de la durée de vie des porteurs, et donnent une mesure de leur durée de vie volumique.
\end{abstract}

\begin{abstract}
Carrier lifetime measurements from photoconductivity (with constant current and constant voltage polarizations of the sample), diffusion photocurrent and open circuit photovoltage responses to transient carrier excitation are compared. Using analytical expressions, the key expressions, conditions of validity and applications of each method are discussed, assuming uniform carrier injection in an unidimensional sample of homogeneous material; the discussion is done taking into account the sample characteristics (width $W$, bulk lifetime $\tau_{v}$, surface recombination velocities $s_{1,2}$, minority carrier diffusion coefficient $D$, equilibrium majority carrier density $p_{0}$ ), excess carrier density $\Delta p$, light pulse duration $T$ and times $t$ of the response considered. The experimental study especially deals with silicon samples up to $W=1 \mathrm{~mm}$ thick, illuminated by $T=2 \mathrm{~ns}$ to $30 \mathrm{~ns}$ laser beams. The responses to penetrating light $\left(\lambda=1.06 \mu \mathrm{m}, \alpha=40 \mathrm{~cm}^{-1}\right.$ in ordinary silicon, $\Delta p=10^{-4}$ to $\left.10^{2} p_{0}\right)$ and strongly absorbed light $\left(\lambda=0.69\right.$ or $0.53 \mu \mathrm{m}, \alpha=2 \times 10^{3}$ and $10^{4} \mathrm{~cm}^{-1}$, beam intensity $E_{0}=10^{-4}$ to $10^{3} \mathrm{~mJ} / \mathrm{cm}^{2}$ ) are used to show the conditions under which the photoresponses observed lead to the same measured values of carrier lifetime and give bulk lifetime measurement.
\end{abstract}

1. Introduction. - The minority carrier lifetime and mobility are the most important factors affecting the conversion efficiency and spectral response of solar cells. And, among them, the minority carrier lifetime is the most sensitive to the cristal defects of the component. This is the reason why special attention is devoted to this electrical parameter in various works applying to solar cells. With this view, we undertook studies on the possibilities of direct carrier lifetime measurements that the transient electrical photoresponses can give, taking into account both the low carrier injection range (where usual measurement methods generally apply) and the higher injection one which is involved in solar cells used under concentrated sunlight.

In a previous paper [1] we already discussed the measurement possibilities which are given by the transient short-circuit current and open-circuit voltage responses to caracterize the minority carrier lifetime in the base and emitter regions of a device. The purpose of the present paper is larger : the study deals with the various transient electrical responses, which are viewed as a whole and with the aim of measuring carrier lifetime in material as well as in device samples. In the following, the conditions of validity and the respective advantages of the photoresponses will thus be compared taking into account (i) the samples caracteristics (resistivity, dimensions, ...) (ii) the carrier excitation level used and (iii) the excitating light wavelength employed for the experiment.

Indeed, the transient electrical photoresponses give a group of methods for measuring the carrier lifetime, in which the carrier lifetime is found directly from the decay (or increase) of voltage or current signals [2]. These photoresponses and associated 
methods are based on either the variation in conductivity produced by both electrons and holes induced in excess in the sample $[3,4]$ - photoconductivity methods using voltage $\left({ }^{1}\right) \Delta V_{\sigma}(t)$ or current $\Delta I_{\Sigma}(t)$ signals depending on whether the sample studied is under constant polarization current or voltage - - or on the diffusion of only the minority carriers induced in excess in the sample - diffusion current method $[5,6]$ or even open-circuit voltage methods [7, 8]. Viewed as a whole, the above methods $\left({ }^{2}\right)$ then may have the advantages of covering various sample structures, including devices at their successive manufacturing stages, and of being utilizable with a single experimental set up.

But, although transient electrical photoresponses tend to be commonly used today for measurements of carrier lifetimes, they are generally used individually, on samples differing in type and/or struature, and under low carrier injection level conditions. Moreover, only for method using the variation in conductivity has the theory been well established for a long time; method using the minority carrier diffusion has been used more recently [1] and is less known especially from the point of view of its conditions of validity for measuring carrier lifetimes.

The following study will thus attempt first of all to compare the key expressions and conditions of validity of each of the above methods, starting with the existing analytical studies of transient photoresponses for uniform carrier injection in an homogeneous sample $[3,6,7,8]$ (Section 2.1). This makes it possible to compare the characteristics of each method considered and to estimate its possibility of application under weak and strong carrier injection conditions (Section 2.2).

In Section 3, the above synthesis is compared with experimental results, in particular results obtained by applying the various methods to the same sample. The experimental study will also attempt to find the limits within which these methods remain applicable in practice, particularly towards high carrier injection conditions and as a function of the coefficient of absorption of the photon pulse. Using the laser pulse excitation technique, the possibilities and limitations of the above methods are illustrated especially in the case of bulk samples of silicon, considering the photoresponses induced by penetrating $1.06 \mu \mathrm{m}$ light pulses and the photoresponses induced by pulses of strongly absorbed $0.53 \mu \mathrm{m}$ or $0.69 \mu \mathrm{m}$ light.

( ${ }^{1}$ ) The photoconductivity method using the $\Delta V_{\sigma}(t)$ signals, or photoconductivity decay technique, is the standard method for measuring carrier lifetime in bulk semiconductors such as germanium and silicon. This standard method is described in the 1971 Annual Book of A.S.T.M. Standard no 8, p. 476.

$\left({ }^{2}\right)$ In this paper, we will not consider (i) the possibility of carrier lifetime measurement by means of transient secondary photocurrent, which may be used for measuring carrier lifetime in the base of a bipolar transistor structure [7]; (ii) the high injection effects on the electrical properties [10].
2. Expressions for transient electrical photoresponses and conditions of validity of the associated carrier lifetime measurement methods. - The electrical photoresponse expressions are obtained by solving the continuity and transport equations of the carriers and by taking into account the sample boundary conditions. The detailed calculations for uniform photoexcitation at weak carrier injection levels are given in references $[3,6]$ and [8] for the transient photoconductivity, diffusion photocurrent and open circuit photovoltage, respectively.

The purpose of the following analysis is to summarize the assumptions on which the electrical photoresponse expressions are based, and to show under what conditions these expressions, which are of the form of a sum of harmonics of order $k$ due to the sample boundary conditions, can be reduced to a rather simple form which can be used as a carrier lifetime method. The key expressions, conditions of validity and applications of the methods are then discussed from a comparative point of view.

2.1 EleCtRICAL PHOTORESPONSE EXPRESSIONS. The electrical photoresponse expressions given below are based on the usual hypotheses concerning semiconductor $\mathbf{N}$ or $\mathbf{P}$ regions : (i) unidimensional, (ii) homogeneous, with negligible electric field, (iii) subjected to a uniform and weak enough carrier injection so as not to alter appreciably the equilibrium majority carrier density, i.e. electric field and average filling rate of the band-gap energy levels, and (iv) characterized by constant values of the $N$ or $P$ region paremeters $W$ (thickness), $\mu_{\mathrm{n}, \mathrm{p}}$ or $D_{\mathrm{n}, \mathrm{p}}$ (electron and hole mobilities or diffusion coefficient), $\tau_{\mathrm{v}}$ (bulk carrier lifetime) and boundary conditions $s_{1,2}$ (surface carrier recombination velocities at $x=0$ and $W$ ).

Because of the transient carrier excitation conditions dealt with in this paper, the photoresponse expressions also assume (v) that the excitation pulse duration $T$ is small [6] compared to the effective carrier lifetime $\tau$ (carrier lifetime taking into account the material bulk carrier lifetime $\tau_{\mathrm{v}}$ as well as the parameters $W, \mu_{\mathrm{n}, \mathrm{p}}, s_{1,2}$ of the $\mathrm{N}$ or $\mathrm{P}$ region), (vi) carrier trapping phenomena $\left({ }^{3}\right)[11,12]$ are negligible in the sample under study, and (vii), if carrier recombination occurs via recombination centers, the recombination center density is small enough $\left({ }^{3}\right)$ so that the neutral and negatively charged recombination center densities remain negligible compared to the total carrier quantities $\left(n_{0}+n_{1}\right)$ and $\left(p_{0}+p_{1}\right)$ respectively in the sample, where the symbols $n_{0}, p_{0}, n_{1}, p_{1}$ have their usual meaning [11].

$\left({ }^{3}\right)$ In all the carrier lifetime measurement methods, injection of excess carries must be accomplished. In all cases, these excess carriers consist of electron-hole pairs produced, in addition to those present at equilibrium, either directly by means of band-toband excitation or due to over-all electrical neutrality following a carrier contact injection. For the transient injection case [11], 
2.1.1 Expressions for conductivity photoresponses. - Let us assume a $P$ tỳpe sample and equilibrium conductivity $\sigma$. The photoconductivity responses $\Delta V_{\sigma}(t)$ and $\Delta I_{\Sigma}(t)$ which refer to constant current and constant voltage conditions (Fig. 1a), respectively, are based on the variation in conductivity $\Delta \sigma(t)$ in the illuminated volume under the effect of $\Delta n(t)=\Delta p(t)$ excess electrons and holes created. The detailed study of $\Delta \sigma(t)$ is done in ref. [3] and gives the following full and simplified expressions :

with [1] :

$$
\Delta \sigma(t)=q \mu_{p}\left[1+\frac{\mu_{n}}{\mu_{p}}\right] \Delta n(t)
$$

$$
\Delta n(t>T)=\Delta \sum_{k=1}^{\infty} R_{k} \exp \left(-t / \tau_{\sigma k}\right)
$$

where $\Delta=\Delta n(t=T)$

If $s_{1} \# s_{2}=s, R_{k}$ and $\tau_{\sigma k}$ are given by :

$$
R_{k}=\frac{\sin ^{2}\left(\xi_{k} \pi / 2\right)}{\xi_{k} \pi\left[\xi_{k} \pi+\sin \xi_{k} \pi\right]}, \quad \frac{1}{\tau_{\sigma k}}=\frac{1}{\tau_{\mathrm{v}}}+\frac{\xi_{k}^{2} \pi^{2} D}{W^{2}}
$$

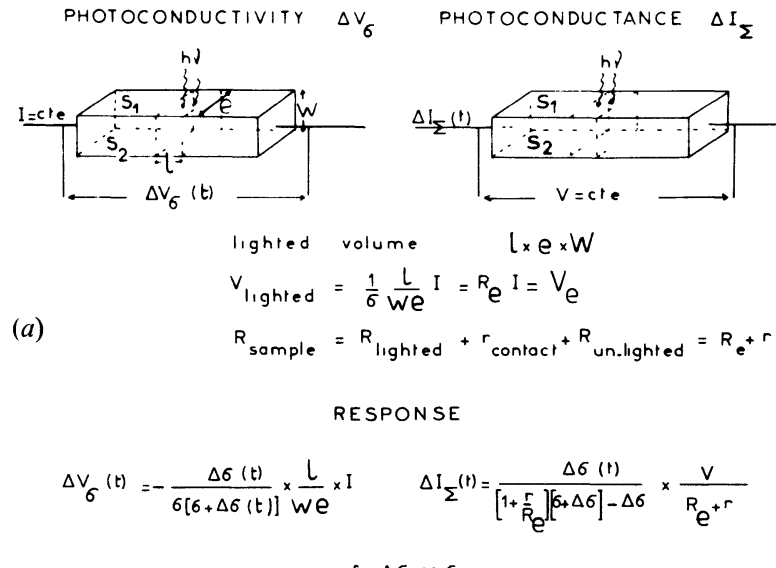

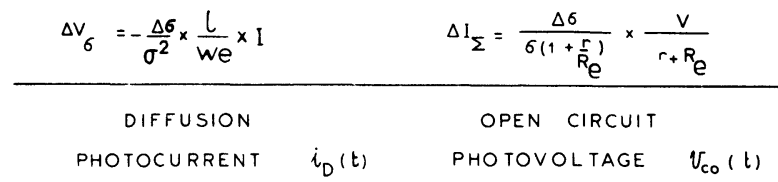

(b)



F 1 g. 1. - Principle of the transient electrical responses of photoconductivity $(a)$, diffusion photocurrent and open circuit photovoltage $(b)$ (equipotential surfaces normal to carrier current flow).

a readjustment time $\tau_{\mathrm{ch}}$ of the charges at the recombination centers is necessary before the conditions of equal capture rate of electrons and holes are satisfied. At times $t>\tau_{\mathrm{ch}}$, the lifetimes of electrons and holes are identical under transient conditions. But only when the hypothesis (vii) is satisfied is $\tau_{\mathrm{ch}}$ negligible compared with electron-hole transient lifetime and is the transient carrier lifetime identical to the minority carrier steady-state lifetime, especially under weak carrier injection.

When trap centers exist in the sample, and if the injected carrie density is not large compared with the trap density, the time that the trapped carriers spend in the traps induces an apparently long decay constant of the excess carriers $[11,12]$. where $D=D_{\mathrm{n}}$ is the minority carrier diffusion coefficient and $\xi_{k}$ is the solution of :

$$
\operatorname{cotg}(\xi \pi / 2)-(\xi \pi D / s W)=0 .
$$

If $s \leqslant 2 D / W$, expression (4) admits the solution $\xi_{k}=2(k-1)$, $k=1,2,3, \ldots$, so that :

$$
\begin{gathered}
R_{1}=1, \quad \frac{1}{\tau_{\sigma 1}}=\frac{1}{\tau_{\mathrm{v}}}+\frac{2 s}{W}=\frac{1}{\tau_{\sigma}}, \\
R_{k}=\frac{s^{2} W^{2}}{2 D^{2}} \frac{1}{(k-1)^{4} \pi^{4}}, \quad \frac{1}{\tau_{\sigma k}}=\frac{1}{\tau_{\mathrm{v}}}+\frac{4 s}{W}+(2 k-2)^{2} \frac{\pi^{2} D}{W^{2}} .
\end{gathered}
$$

The harmonics of order $k>1$ therefore have an amplitude $R_{k}$ and time constant $\tau_{\sigma k}$ very small compared to $R_{1}$ and $\tau_{\sigma}$, and decreasing as $k$ increases. The same is true if $s \gg 2 D / W$, a condition which includes $\xi_{k}=2 k-1$, with $R_{2} / R_{1}$ and $\tau_{\sigma 2} / \tau_{\sigma 1} \# 1 / 9$.

Expression (1) for $\Delta \sigma(t)$ can therefore be written in the form :

$$
\Delta \sigma(t>T)=q \mu_{p} \Delta\left[1+\frac{\mu_{n}}{\mu_{p}}\right] \exp \left(-t / \tau_{\sigma}\right)
$$

if $T \ll \tau_{\sigma}, s_{1} \# s_{2}=s$ with

and

$$
\begin{gathered}
\frac{1}{\tau_{\sigma}}=\frac{1}{\tau_{\mathrm{v}}}+\frac{2 s}{W} \text { if } s \ll 2 D / W \\
\frac{1}{\tau_{\sigma}}=\frac{1}{\tau_{\mathrm{v}}}+\frac{\pi^{2} D}{W^{2}} \quad \text { if } \quad s \gg 2 D / W .
\end{gathered}
$$

2.1.2 Expressions for diffusion photocurrent $i_{\mathrm{D}}(t)$. - Only the minority carriers $\Delta n(t)$ from illuminated neutral regions are concerned in this photoresponse due to the carriers'ability to reach and cross a collection zone (a zone which is equivalent to a large recombination velocity $s_{1}$ boundary $\left({ }^{4}\right)$ ), such as an $\mathrm{n}^{+} \mathrm{p}$ junction (Fig. $1 b$ ).

Unlike the previous photoresponses based on the conductivity variation, the $i_{\mathrm{D}}(t)$ response involves a major contribution from the $k>1$ harmonics $[6]\left({ }^{4}\right)$. Because of this contribution, the expression for $i_{\mathrm{D}}(t)$ may, for large enough $s_{1}$, be written as :

$$
i_{\mathrm{D}}(t)=q A \Delta \frac{2 D}{W} \sum_{k=1}^{m} \exp \left(-t / \tau_{i k}\right)
$$

when $t>3 T, T<\frac{1}{3} \tau_{i, k=m}$ with

$$
\frac{1}{\tau_{i k}}=\frac{1}{\tau_{\mathrm{v}}}+X_{k}^{2}
$$

$X_{k}$ depends on the recombination velocity $s_{2}$ on the back side :

$$
\begin{aligned}
& X_{k}^{2}=(2 k-1)^{2}\left(\pi^{2} D / 4 W^{2}\right) \text { when } s_{2}=0 \\
& X_{k}^{2}=(2 k-1)^{2}\left(\pi^{2} D / W^{2}\right) \quad \text { when } \quad s_{2}=\infty .
\end{aligned}
$$

Depending on whether the values of the product $t X_{k=1}^{2}$ are large or small with respect to one, expression (10) is reduced to either of the two following simplified forms [6] :

$$
\begin{gathered}
i_{\mathrm{D}}\left(t>t_{1}\right)=q A \Delta \frac{2 D}{W} \exp \left(-t / \tau_{i}\right), \\
\tau_{i}=\tau_{i, k=1}, \quad t_{1}=X_{1}^{-2}
\end{gathered}
$$

$$
i_{\mathrm{D}}\left(3 T<t<t_{1}\right)=q A \Delta \sqrt{\frac{D}{\pi}} \frac{1}{\sqrt{t}} \exp \left(-t / \tau_{\mathrm{v}}\right) .
$$

(4) With respect to reference [6], $s_{1}=\beta v_{\mathrm{D}}, \Delta=T G$ and $X_{k}^{2}=Z_{k}^{2} / \tau_{v}$.

From the study of reference [6], the given expressions for $i_{\mathrm{D}}(t)$ appear valid as soon as $\beta$ is large compared with unity, i.e., $s_{1}$ large compared with the minority carrier diffusion velocity $v_{\mathrm{D}}$ inside the sample. 
2.1.3 Expressions for open-circuit photovoltage $v_{\mathrm{co}}(t)$. - The open-circuit photovoltage responses $v_{\mathrm{co}}(t)$ developed across the collecting zone obey the collecting zone current equation :

where

$$
I_{\mathrm{h} v}(t)+I\left(V_{\mathrm{A}}\right)+I_{\mathrm{c}}\left(V_{\mathrm{l}}\right)=0
$$

and

$$
V_{\mathrm{A}}=V_{\mathrm{Ao}}+v_{\mathrm{co}}(t), \quad V_{\mathrm{I}}=v_{\mathrm{co}}(t)-\left|V_{\mathrm{Io}}\right|
$$

$$
I_{\mathrm{c}}\left(V_{\mathrm{l}}\right)=C \frac{\mathrm{d} V_{\mathrm{I}}}{\mathrm{d} t}+V_{\mathrm{I}} \frac{\mathrm{d} C}{\mathrm{~d} t} .
$$

Here, $I_{\mathrm{hv}}(t)$ is the total induced photocurrent, due to the excess carriers which can be created both in the neutral regions (i.e. diffusion current) and in the collecting zone itself. $V_{\mathrm{Ao}}$ and $V_{\mathrm{Io}}$ are the equilibrium applied voltage and collecting zone voltage, respectively, and $C$ is the collecting zone capacity $C\left(V_{\mathrm{l}}\right)$. In the case where the collecting zone is a pn junction, $I\left(V_{\mathrm{A}}\right)$ is given exactly by :

$$
I\left(V_{\mathrm{A}}\right)=I_{0}\left[\exp \left(\frac{q V_{\mathrm{A}}}{k T}\right)-1\right]
$$

where $I_{0}$ is a constant.

The analytical expressions of the transient photoresponse $v_{\mathrm{co}}(t)$ are discussed in ref. [8]. This study shows that in this type of photoresponse the collection zone time constant and the minority carriers of the two regions bordering this zone play an important part. However, for just this reason, the analytical expressions for $v_{\mathrm{co}}(t)$ remain cumbersome. As the purpose of the present study is to compare the conditions under which the photoresponses may be reduced to simpler forms to provide a method for measuring carrier lifetime, the following study will consider the photoresponses in those cases which lend themselves to a simplified formulation [1].

2.1.3.1 Under the effect of $I_{\mathrm{hy}}(t)$. - Expression (16) shows that the induced response $v_{\text {co }}(t)$ increases and is only described by a simple expression if, at the times $t$ considered, the conditions below are satisfied :

and

$$
I\left(V_{\mathrm{A}}\right) \ll I_{\mathrm{c}}\left(V_{\mathrm{l}}\right)
$$

$$
I_{\mathrm{c}}\left(V_{\mathrm{I}}\right) \# C(1-K) \frac{\mathrm{d} V_{\mathrm{I}}}{\mathrm{d} t}
$$

where $K$ is a constant.

At times $t>t^{\prime}$ where the diffusion of a single type of carrier dominates, the $v_{\text {co }}(t)$ responses then obey :

$$
v_{\mathrm{co}}\left(t>t^{\prime}\right)=v_{\mathrm{co}}\left(t^{\prime}\right)+\frac{1}{C(1-k)} \int_{t^{\prime}}^{t} i_{\mathrm{D}}(y) \mathrm{d} y \quad \forall V_{\mathrm{Ao}}
$$

where $i_{\mathrm{D}}\left(y>t^{\prime}\right)=I_{\mathrm{h} v}\left(y>t^{\prime}\right)$ is given by (14) or (15).

From (22), the increase in $v_{\text {co }}(t)$ is then, under the conditions in expression (14) i.e. if long enough times $t$ are considered :

$$
\begin{aligned}
v_{\mathrm{co}}\left(t>t^{\prime} \geqslant t_{1}\right)=v_{\mathrm{co}}\left(t^{\prime}\right)+\left[v_{\mathrm{co}}\left(t_{\infty}\right)-v_{\mathrm{co}}\left(t^{\prime}\right)\right] \times \\
\left.\times\left[1-\exp -\left(t-t^{\prime}\right) / \tau_{i}\right)\right] .
\end{aligned}
$$

When $i_{\mathrm{D}}\left(y>t^{\prime}\right)$ is given by (15), only the variation with $t$ of the derivative of the expression (22) may be reduced to a simple form, if short enough times $t$ are considered :

$$
\frac{\mathrm{d} v_{\mathrm{co}}(t)}{\mathrm{d} t}\left(3 T, t^{\prime}<t<t_{1}\right) \quad \text { cf. }(15)
$$

2.1.3.2 When $I_{\mathrm{h} v}$ is zero. - The simplest way of studying the phenomena which may be involved in the decay of $v_{\text {co }}(t)$ is to use the charge controlled model. This model gives a quasistatic approximation valid in first order. Expression (16) then becomes

with

$$
I\left(V_{\mathrm{A}}\right)=-\mathrm{d} Q / \mathrm{d} t
$$

$$
Q=Q_{\mathrm{N}^{+}}+Q_{\mathrm{P}}
$$

where $Q_{\mathrm{N}^{+}}$and $Q_{\mathrm{P}}$ represent the charges stored on either side of the collection zone.

Such a study is done in detail in reference [5] for the case where a collection zone can be assimilated with an ideal p-n junction. This implies in particular that (i) the carrier recombination is negligible both inside the collecting zone and on its surface channels and (ii) the collecting zone behavior is dominated by carrier diffusion processes such as $I\left(V_{\mathrm{A}}\right)$ is described by expression (19) with

$$
\begin{aligned}
& I_{0}=\frac{Q_{\mathrm{N}^{+} \mathrm{o}}}{\tau_{\mathrm{N}^{+}}}+\frac{Q_{\mathrm{Po}_{\mathrm{o}}}}{\tau_{\mathrm{P}}} \\
& Q=\left[Q_{\mathrm{N}^{+} \mathrm{o}}+Q_{\mathrm{Po}^{\circ}}\right]\left[\exp \left(\frac{q V_{\mathrm{A}}}{k T}\right)-1\right]
\end{aligned}
$$

$Q_{\mathrm{N}^{+} \mathrm{o}}$ and $Q_{\mathrm{Po}_{\mathrm{o}}}$ are the reference charges of the $\mathrm{N}^{+}$and $\mathrm{P}$ regions on either side of the collection zone. The lifetimes $\tau_{\mathbf{N}^{+}}$and $\tau_{\mathbf{P}}$ in expression (27) are the operative lifetimes of the minority carriers of the $\mathrm{N}^{+}$and $\mathbf{P}$ regions, and may take into account the various phenomena which can intervene in this minority carrier transport, e.g., the high doping and doping profile effects in a diffused $\mathrm{N}^{+}$ region.

For $V_{\mathrm{A}} \gg k T / q=U_{\mathrm{T}}$, expressions (19), (27), and (28) then lead to a linear $v_{\mathrm{co}}(t)$ decay of the form :

where

$$
\begin{gathered}
v_{\mathrm{co}}(t)=v_{\mathrm{comax}}-U_{\mathrm{T}} t / \tau_{\mathrm{c}} \quad \forall V_{\mathrm{Ao}} \\
\tau_{\mathrm{c}}=\left[Q_{\mathrm{N}^{+} \mathrm{o}}+Q_{\mathrm{Po}^{\circ}}\right] /\left[\frac{Q_{\mathrm{N}^{+} \mathrm{o}}}{\tau_{\mathrm{N}^{+}}}+\frac{Q_{\mathrm{Po}_{0}}}{\tau_{\mathrm{P}}}\right] .
\end{gathered}
$$

However, when $V_{\mathrm{A}} \# v_{\mathrm{co}}(t) \ll U_{\mathrm{T}}$, the $v_{\mathrm{co}}(t)$ response decreases exponentially according to :

$$
v_{\text {co }}(t)=v_{\text {comax }} \exp \left(-t / \tau_{\mathrm{c}}\right) \text {. }
$$

\subsection{COMPARED ASPECTS OF THE CARRIER LIFETIME} MEASUREMENT METHODS USING TRANSIENT PHOTORESPONSES. - The above analysis makes it possible to see the conditions of validity and key expressions on which the carrier lifetime measurement methods using the transient photoresponses are based. Assuming hypotheses (i) through (vii) are satisfied :

a) Expressions (7), (8) and (9) indicate that the decrease in conductivity responses (Fig. 1a) is governed by a time constant $\tau_{\sigma}$. This lifetime $\tau_{\sigma}$ is essentially a function of parameters $\tau_{\mathrm{v}}, W, s_{1,2}$ of the sample, and last $\tau_{\mathrm{v}}$ if $W / 2 s \gg \tau_{\mathrm{v}}$ when $s_{1} \not \# s_{2}=s$.

b) The diffusion photocurrents lend themselves to the measurement of the carrier lifetime when they can be described by expressions (14) or (15) : depending on the time $t$ of the decrease of the responses considered, and depending on the parameters $D, W$, $\tau_{\mathrm{v}}, s_{1}, s_{2}$ of the diffusion region studied, expressions (14) and (15) therefore show that the lifetimes $\tau_{i}$ or $\tau_{v}$ are given by the time constant of the response $i_{\mathrm{D}}\left(t>t_{1}\right)$ or the time constant of the product $i_{\mathrm{D}}\left(3 T<t<t_{1}\right) \cdot \sqrt{t}$, respectively [6]. In addition, it should be noted that the use of expressions (14) and (15) above assume that the total current $I_{\mathrm{h} v}(t)$ given by the collection zone is, at the times $t>t^{\prime}$ studied, dominated by the diffusion of excess minority carriers from a single edge region

$$
\left(\left(i_{\mathrm{DN}}+\left(t>t^{\prime}\right) \ll i_{\mathrm{DP}}\left(t>t^{\prime}\right),\right.\right.
$$


in the example in figure $1 b$, if carrier lifetime of the $\mathbf{P}$ region is considered, for example) as discussed in ref. [1].

c) Except in some cases, and thus unlike the cases of the previous photoresponses, the open-circuit photovoltage responses can lead to unreliable measurements of the carrier lifetime in a given sample neutral region, especially when they are used alone. However, the transient photovoltage may present specific applications, particularly for $\mathrm{N}^{+}-\mathrm{P}$ structure samples [1], either because the photovoltage response decay permits measurements which are complementary (see also [7]) to those obtained using diffusion photocurrents (for example, evaluation of $\tau_{N^{+}}$using $\tau_{c}$, if $\tau_{\mathrm{P}}$ is given by $i_{\mathrm{DP}}(t)$ or vice versa), or because, for the light pulse used, the increase and the decay of a single photovoltage response are each dominated by a different region, i.e., $\tau_{\mathbf{N}^{+}}$and $\tau_{\mathbf{P}} \# \tau_{\mathrm{c}}$ for example [1].

Concerning the conductivity $\Delta V_{\sigma}(t)$ or $\Delta I_{\Sigma}(t)$ and diffusion current $i_{\mathrm{D}}(t)$ photoresponses, their additional major properties, from the point of view of their experimental use, can be summarized as follows :

d) All these responses show a linear increase with the injected carrier level $\Delta$ insofar as the carrier mobilities $\mu_{n, p}$ are independent of $\Delta$. The response maximum corresponds to times $t_{\max }=T$ when the excitation pulse $G(t)$ is rectangular $G(t \leqslant T)=G$, $\Delta=G T$; it corresponds to times $t_{\max }=2 T$ and $T<t_{\max } \leqslant 2 T$ in the case of a triangular pulse of half-width duration $T$ for the $\Delta V_{\sigma}(t)$ or $\Delta I_{\Sigma}(t)$ and $i_{\mathrm{D}}(t)$ responses [6], respectively.

e) Depending on the sample studied, they can lead to various measurement sensitivities in the lowest $\Delta$ range (Fig. 1a) : the responses $\Delta V_{\sigma}(t)$ and $\Delta I_{\Sigma}(t)$ vary as $\sigma^{-2}$ and $[\sigma(1+r / \operatorname{Re})]^{-1}$ respectively, i.e., either strongly depend on the sample conductivity $\sigma$ or depend on both $\sigma$ and the sample contact resistances in $r$. The $i_{\mathrm{D}}(t)$ response sensitivity only varies with the sample minority carrier mobility $\mu_{\mathrm{n}}$; it can therefore be better than the sensitivity of the responses $\Delta V_{\sigma}(t)$ and $\Delta I_{\Sigma}(t)$ in strong conductivity samples. The same is true for the response sensitivity $v_{\text {co }}(t)$.

$f)$ The time constants $\tau_{\sigma}$ and $\tau_{\mathrm{i}}$ are not identical. For thin neutral regions, the conductivity responses then usually afford better possibilities than the $i_{\mathrm{D}}$ responses do (cf. expressions (7), (8), (9) and (14), (15), (12), (13)).

g) Moreover, it should be noted that, when the excess carrier density $\Delta$ is increased up to $\Delta$ values around the equilibrium majority carrier density $p_{0}$, the expressions (7), (14) and (15) can again be used to describe the photoresponses around a given time $t$, provided the variations of the sample parameters $\mu_{\mathrm{n}, \mathrm{p}}$ or $D_{\mathrm{n}, \mathrm{p}}, \tau_{\mathrm{v}}$ and $s_{1,2}$ with $\Delta$ and the time $t$ are taken into account $[4,6]$. In this $\Delta$ range however, the photocurrent $i_{\mathrm{D}}(t)$ decay method given by (14) or
(15) may appear more precise than those for the photoconductivity decay : the expressions for $\Delta V_{\sigma}(t)$ and $\Delta I_{\Sigma}(t)$, as given in figure $1 a$, are then more complicated functions of $\tau_{\sigma}$ than (7). But, as $\Delta$ increases above $p_{0}$, the expression (7) is again likely to describe the photoconductivity response decay. In this $\Delta>p_{0}$ case, it can be noted that the responses $i_{\mathrm{D}}(t)$ lend themselves even better to measuring the carrier lifetime as this measurement can be made near the start of the decay (cf. (15)) and thus lowers the effects of the variations $D(x, t), \tau_{\mathrm{v}}(x, t)$ with the induced density $n, p(x, t)$ of carriers present in $x$ at the time $t$.

3. Experimental results. - The purpose of the following experimental data is to compare the results obtained from the methods analyzed in section 2, and to study the hypotheses (i) to (vii) involved in the analysis of section 2. For this reason, the reported results deal particularly with P-type silicon monocrystalline samples, of $\sim 1$ and $10 \Omega$.cm, even though this type of sample does not lend itself to illustrating the various possibilities that diffusion current $i_{\mathrm{D}}(t)$ and voltage $v_{\text {co }}(t)$ photoresponses can have in a device [1]. The first part of the results is then devoted to the comparison of the photoresponses of conductivity $\Delta V_{\sigma}(t)$ and $\Delta I_{\Sigma}(t)$, diffusion current and opencircuit voltage induced, with the aid of a $1.06 \mu \mathrm{m}$ penetrating light pulse, over a wide range of carrier injection level $\Delta$. The second part of the results aims to illustrate the effects that the light absorption coefficient have on the transient electrical photoresponses and on their associated possibilities in measuring carrier lifetime. This deals especially with the shape and decay of bulk sample photoresponses measured, depending on the sample illuminated face, when using a smoothly and a strongly absorbed light pulse. The corresponding studies and results are illustrated in figures 2 to 4 .

The surfaces of the monocrystalline samples studied (Fig. 2) are carefully cleaned ( $s$ small), and the sample length and width are chosen large enough compared to the sample thickness so that the samples studied can be considered as unidimensional (cf. hyp. (i)). For the photoconductivity measurements, the polarisation values $V$ and $I$ are also chosen so that the transit time of the excited carriers, from their site of excitation to one of the sample contacts, remains large compared to the decay of the measured signal, i.e., signal decay independent of $V$ or $I$, cf. hyp. (ii). The responses $i_{\mathrm{D}}(t)$ may be studied with a reverse bias on the collecting contact to ensure a high enough value $s_{1}$ during the measurements (cf. (10)).

The experimental set up consists of a CILAS VNDM 20 laser (2 $T=70 \mathrm{~ns}$ ) with a neodymium doped $\left(\lambda=1.06 \mu \mathrm{m}, E_{0 \max }=1 \mathrm{~J}\right)$ or ruby $(\lambda=0.69 \mu \mathrm{m})$ rod and a CILAS VD 162 laser with a neodymium doped $\operatorname{rod}\left(2 T=55 \mathrm{~ns}, E_{0 \max }=3 \mathrm{~J}\right)$. The latter may be associated with an electro-optic shaping cell CILAS DL 11, which can reduce the laser pulse duration to $T=2 \mathrm{~ns}$, and to a frequency-doubling crystal $(\lambda=0.53 \mu \mathrm{m})$. Measurements are performed inside a $8 \mathrm{~m}^{3}$ Faraday cage of $50 \mathrm{~dB}$ attenuation at $1 \mathrm{GHz}$, and with a digital transient signal analyzer (Tektronix WP 22 21) including a R 7912 transient digitizer and a PDP 11 calculator with $16 \mathrm{k}$ words of core memory. A 1 to $6 \mathrm{~mm}$ diameter optical fiber conveys the laser beam to the sample which is in the dark. At the fiber input, calibrated attenuators are used to adjust the light 
(a)



(b)



Fig. 2. - Monocrystalline (P-type silicon) samples used for the comparative studies of the electrical responses of photoconductivity $(a)$, and diffusion photocurrent or open circuit photovoltage $(b)$. (1) = aluminium cold-deposition, (2) = boron cold-implanted layer, (3) = light-mask, (4) = illuminated area, (5) = front illumination, (6) = back illumination.
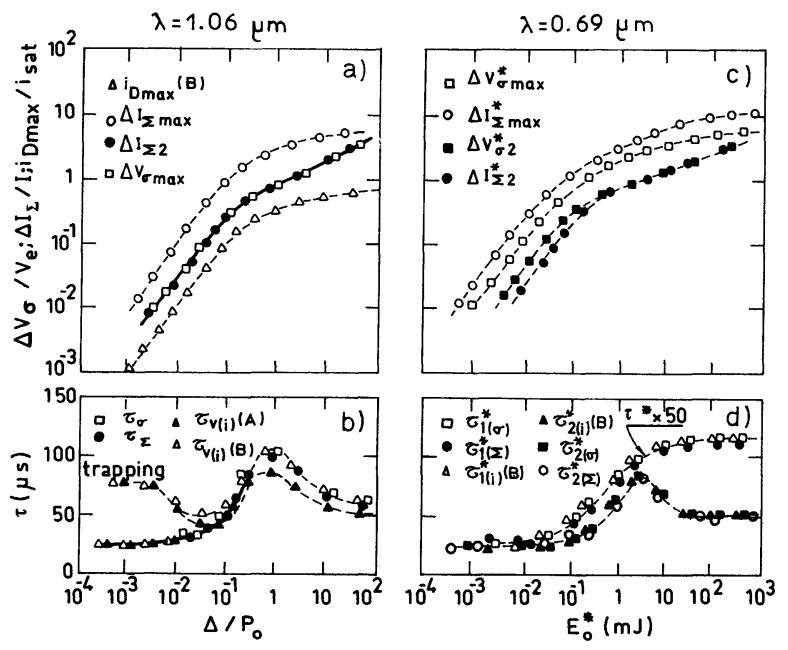

Fig. 3. - Evolution versus $\Delta$ - or pulse intensity $E_{0}^{*}$ - of the measured amplitudes and decay time constants for the photoresponses of $\mathbf{P}$ type monocrystalline silicon sample submitted to front illumination (cf. Fig. 2) by $2 T=70 \mathrm{~ns}$ laser pulses at $1.06 \mu \mathrm{m}$ (left hand : Fig. $3 a, b)$ and $0.69 \mu \mathrm{m}(*$; right hand : Fig. $3 c, d$ ). Sample : $0.8 \Omega . \mathrm{cm}, \mathrm{B}$ doped, floating zone, EPD : 20 to $35 \times 10^{3} \mathrm{~cm}^{-2}$. Solid curve in figure $3 a$ is the photoconductivity amplitude as given in figure $1 a$ when taking into account the mobility variations $\mu_{\mathrm{n}, \mathrm{p}}\left(N=p_{0}+\Delta\right)$. Solid curve in figure $3 b$ assumes a usual Hall-Schockley-Read one-level recombination lifetime.

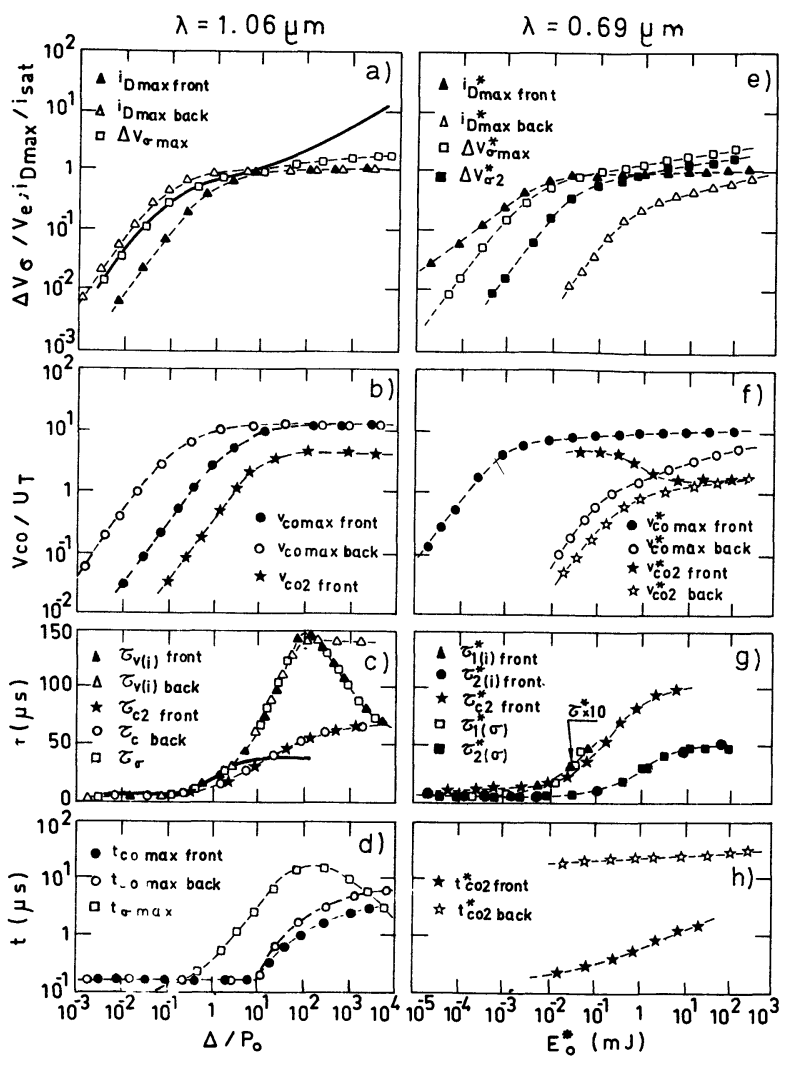

Fig. 4. - Evolution versus $\Delta$ - or pulse intensity $E_{0}^{*}$ - of the measured amplitudes, decay-time constants and descriptive times for the photoresponses of $P$ type monocrystalline silicon sample submitted to front and back illumination (cf. Fig. 2) by $2 T=70 \mathrm{~ns}$ laser pulses at $1.06 \mu \mathrm{m}$ (left hand : Fig. $b, c, d)$ and $0.69 \mu \mathrm{m}\left({ }^{*}\right.$; right hand : Fig. $4 e, f, g, h)$. Sample : $9.2 \Omega$. cm, Al doped, floating zone, EPD : 20 to $35 \times 10^{3} \mathrm{~cm}^{-2}$. Solid curve in figure $4 a$ is the photoconductivity amplitude as given in figure $1 a$ when taking into account the mobility variations $\mu_{\mathrm{n}, \mathrm{p}}\left(N=p_{0}+\Delta\right)$. Solid curve in figure $4 c$ assumes a usual Hall-Schockley-Read one-level recombination lifetime.

exciting pulse intensity $E_{0}$ on the sample. The laser pulse intensity on the attenuators is monitored by a PIN photodiode CG 530 whose response is calibrated with a CILAS PS 16 calorimeter. The photodiode response is used to trigger the sample signal measurement. A band-pass optical filter $(\Delta \lambda=10 \mathrm{~nm})$ is placed at the optical fiber input when the $1 \mathrm{~ms}$ duration light from the xenon flash of laser pumping is to be eliminated.

\subsection{PhOtORESPONSE COMPARISON (PENETRATING} LIGHT PULSE). - The results obtained with the usual monocrystalline silicon samples when using the penetrating $1.06 \mu \mathrm{m}$ laser beam are illustrated in figure $3 a$, $b$ and $4 a$ to $d$.

3.1.1 For excess carrier density $\Delta \lesssim p_{0}$. - The amplitude and decay time of the measured photoresponses of conductivity $\Delta V_{\sigma}(t)$ and diffusion current $i_{\mathrm{D}}(t)$ agree with the expressions of figure $1 a$ and (7), (8) and (10), (15), respectively. In particular, the measured decay time $\tau_{\sigma}$ corresponds to the bulk lifetime $\tau_{\mathrm{v}}$ deduced from $i_{\mathrm{D}}(t)$ by using the $i_{\mathrm{D}} \cdot \sqrt{t}$ product decay - cf. $2.2, a), b$ ) comments applied to a sample with large $W$ and small $s$. It is to be noticed 
here that, in practice, some discrepancy may appear between the measured values $\tau_{\sigma}, \tau_{\mathbf{v}(\mathbf{i})}$ due to the illuminated sample regions involved : e.g., in figure $3 b$, $\tau_{\mathrm{v}(\mathrm{i})}(\mathrm{A})$ and $\tau_{\mathrm{v}(\mathrm{i})}(\mathrm{B})$ slightly different values are obtained by using sample contacts $A$ and $B$, respectively. For the lowest $\Delta$, temporary minority carrier trapping may induce an increase in the measured lifetime; such an effect is shown in figure $3 b$ which, moreover, illustrates the great sensitivity of the $i_{\mathrm{D}}(t)$ responses for studying low resistivity samples - cf. $2.2, e$ ) comment. The trapping effect is then eliminated when the sample is submitted to a pseudo-steadystate light pulse (the laser exciting xenon flash in the measurements of figure $3 b$; cf. hyp. (vi)). When $\Delta$ increases, the $\tau_{\mathrm{v}(\mathrm{i})}(\Delta)$ variation in figures $3 b, 4 c$ agree with the variation of a usual Hall-ShockleyRead one-level-recombination lifetime. It can also be noted that the increase of the amplitude with $\Delta$ (cf. Fig. $3 a, 4 a$ ) and the amplitude times $t_{\max }$ of the diffusion photocurrent and photoconductivity signals agree with comment $2.2, d$ ); in particular, the decay $\Delta V_{\sigma}(t)$ obeys (7) as soon as $t \geqslant 2 T$ in the range $\Delta \lesssim p_{0}$ (cf. Fig. $4 d$ ). Hypothesis (iv), (v) and (vii) are thus also satisfied in the studied samples for the transient carrier injection conditions used. Furthermore, for $\Delta<10^{-1} p_{0}$ and thus $\Delta \sigma \ll \sigma$, the $\Delta$ values deduced from the measured photoconductivity signal amplitudes $\Delta V_{\sigma \max }$ (cf. Fig. 1a) agree well with the values calculated from the intensity $E_{0}$ of the incident $1.06 \mu \mathrm{m}$ light pulse when assuming a $20 \%$ light reflection at the sample surface, a quantum efficiency of unity $\left(\Delta \propto E_{0}\right)$ and a homogeneous absorption of the light in the sample thickness $W=1 \mathrm{~mm}$. When $\Delta$ values approach $p_{0}$ such that

$$
0.3 p_{0}<\Delta \leqslant 3 p_{0}
$$

only an average decay time constant $\left\langle\tau_{\sigma}\right\rangle$ is found to describe the photoconductivity response decay, even when measuring this decay-time towards the beginning of the decay. This is in agreement with the decay expression on $\Delta V_{\sigma}(t)$ in this $\Delta$ range - cf. comment $2.2, g)$. However, the measured average decay time $\left\langle\tau_{\sigma}\right\rangle$ remains in agreement with the decay time $\tau_{\mathrm{v}(\mathrm{i})}$ given by the responses $i_{\mathrm{D}}(t)$ as shown in figures $3 b$ and $4 c$.

In contrast to the above observed signals $\Delta V_{\sigma}(t)$ and $i_{\mathrm{D}}(t)$, the photoconductivity signals $\Delta I_{\Sigma}(t)$ measured when using constant voltage condition appear as the sum of two responses (cf. Fig. 3a) :

$$
\Delta I_{\Sigma}(t)=\Delta I_{\Sigma 1}(t)+\Delta I_{\Sigma 2}(t) .
$$

Only the amplitude and decay of the slowest component $\Delta I_{\Sigma 2}(t)$ then agree with the sample bulk photoconductivity response (7), i.e. agree with the values and variations with $\Delta$ of the amplitude and decay of the $\Delta V_{\sigma}(t)$ responses. In the case of figure 3, the $\Delta I_{\Sigma 1}(t)$ component shows a decay time constant $\tau_{\Sigma 1} \# 0.8 \mu \mathrm{s} \forall E_{0}$. This fast component varies with sample surface treatment and appears as an enhanced variation of the equilibrium current density in the sample region near the surface. The measured signals $v_{\text {co }}(t)$ may also appear as the sum of two responses (cf. Fig. 4b). However, the signals obtained for a front illumination must be distinguished here from those obtained for a back illumination of the sample (Fig. 2b). In particular, only the front illumination signals, as shown in figure $4 b, c$, decay as the sum of two responses :

$$
v_{\text {cof ront }}(t)=v_{\text {co1 }}(t)+v_{\text {co2 }}(t) .
$$

The back illumination signals are reduced to the slowest component above, $v_{\mathrm{co} 2}(t)$. The fast component $v_{\mathrm{co} 1}(t)$ would thus give evidence of a sample surfacecontact contribution for collecting excess carriers. Due to the increased lighted area of the sample - cf. figure 2-the amplitude of the diffusion current $i_{\mathrm{D} \max }$ and open circuit voltage $v_{\mathrm{co}}(t)$ signals appear higher for the back illumination of the sample (cf. Fig. $4 a, b$ ). The value of the results illustrated in figure $4 c$ is to show that the measured decay time constant $\tau_{\mathrm{c} 2}$ of the $v_{\mathrm{co} 2}$ front illumination component, and the decay time constant $\tau_{c}=\tau_{c 2}$ of the back illumination response, may agree with the measured decay time $\tau_{\sigma}=\tau_{\mathbf{v}(\mathbf{i})}$. The agreement is observed as long as the amplitude of the photovoltage component involved is small compared with $U_{\mathrm{T}}\left(\mathrm{cf}\right.$. (31), $V_{\mathrm{Ao}}=0$ ). As shown in figure $4 d$, the rise time $\sim t_{\text {comax }}$ of the photovoltage signals remains small - cf. comment $2.2, c)$ for the case $\tau_{\mathrm{c}}=\tau_{\mathrm{P}}$ -

If we except the enhanced near surface contributions $\Delta I_{\Sigma 1}(t)$ and $v_{\mathrm{co} 1}(t)$, the above results thus show that the photoconductivity, diffusion-photocurrent and also open-circuit photovoltage decay methods described in section 2 lead to the same (bulk) carrier lifetime measured values $\tau_{\sigma, \mathrm{v}(\mathbf{i}) \mathrm{c}}=\tau_{\mathrm{v}}$.

3.1.2 In the high excitation range $\Delta>p_{0}$. - The instantaneous photoresponse decay is used to measure the carrier lifetime. The carrier lifetime values measured at the decay beginning of the signals $\Delta V_{\sigma}(t)$ - response in the standard carrier lifetime measurement method,$- \Delta I_{\Sigma 2}(t)$, and $i_{\mathrm{D}}(t)$ by using (15), remain in good agreement as figures $3 b$ and $4 c$ show. On the other hand, the decay time $\tau_{c}$ of the photovoltage response decay $v_{\mathrm{co}}(t)$ differs increasingly with the previously measured carrier lifetime as $\Delta$ increases; the difference between the excited carrier density $\Delta$ and the carrier level effective in the open circuit photovoltage, and between the relative values of $v_{\mathrm{co}}(t)$ and $U_{\mathrm{T}}$ in the sample studied, could explain the measured values of $\tau_{\mathrm{c}}$. As a whole, the above results agree with the remarks in section $2.2, \mathrm{~g}$ ).

It should also be noted that, in this $\Delta$ range, the photocurrent signal amplitude $i_{\mathrm{D} \max }$ always tends to saturate at the value $i_{\text {sat }}=v_{\text {cosat }} / Z_{\text {tot }}$ where $Z_{\text {tot }}$ is the total circuit impedance and $v_{\text {cosat }}$ the measured maximum amplitude [13] of the $v_{\text {co }}(t)$ collecting zone 
response. On the other hand, the signal amplitudes $\Delta V_{\sigma \max }$ and $\Delta I_{\Sigma 2}$ either continue to increase with $\Delta$ (cf. Fig. 3a) or saturate (cf. Fig. 4a) above the maximum value of the response amplitude such as $V_{\mathrm{e}}$, calculated from the sample equilibrium parameters $\mu_{\mathrm{n}, \mathrm{p}}, p_{0}, V$ or $I$ (Fig. $1 a$ ). For the case shown in figure $3 a$, the usual case for $1 \Omega$.cm P-type silicon, the observed increase agrees with the theoretical decrease in majority carrier mobility $\mu_{\mathrm{p}}(\Delta)$ that would be in accord with a total impurity concentration $N=p_{0}+\Delta[14]$. A simultaneous decrease of the lifetime values is observed (cf. Fig. $3 b$ ) which qualitatively agrees with the surface recombination velocity variation $s=s_{0}\left(1+\Delta / p_{0}\right)$ from $s_{0}$ to $s_{\infty}$ (cf. (8), (9) for example; $s_{0}=200 \mathrm{~cm} / \mathrm{s}$ in figure $3 b$ ). In the results of figures $4 a, b, c$, alterations in the average level filling rates (cf. hyp. (iii)) should be involved to explain both the response amplitudes and the carrier lifetime behavior observed [2]. Moreover, due to such carrier injection effects, it is to be noted that the amplitudes of the observed $\Delta V_{\sigma}(t)$ and $\Delta I_{\Sigma 2}(t)$ responses usually appear at times $t_{\text {max }}$ large compared with $2 T$ when $\Delta$ is greater than $10^{-1} p_{0}$ (cf. Fig. $4 d$ ).

3. 2 LIGHT ABSORPTION COEFFICIENT EFFECTS. - The influences of the light absorption coefficient value $\alpha$ and of the associated sample thickness where carrier excitation occurs (cf. hyp. (iii)) are illustrated in two ways in the results in figures 3 and 4 . First, as previously noted in paragraph 3.1 , these results show a good agreement between the values $\tau_{\mathrm{v}}(\Delta)$ given by the $i_{\mathrm{D}}(t)$ and $v_{\text {co }}(t)$ responses which are obtained when lighting the sample with the $1.06 \mu \mathrm{m}$ pulses either on the surface near the contact or on the opposite surface of the sample (cf. Fig. 4c). For carrier lifetime measurements, excitation by $1.06 \mu \mathrm{m}$ light in ordinary silicon $\left(\alpha=30-40 \mathrm{~cm}^{-1}\right)$ up to $1 \mathrm{~mm}$ thick thus appears to give a good experimental condition of uniform carrier injection. The second illustration is given by the comparison of the sample photoresponses obtained with the $1.06 \mu \mathrm{m}$ pulses, and with the strongly absorbed $0.69 \mu$ m ones $\left(\alpha \# 2.5 \times 10^{3} \mathrm{~cm}^{-1}\right.$; cf. figure $3 c, d$, $4 e$ to $h)$. In the latter case, all the measured sample photoresponses show two components. For any $0.69 \mu \mathrm{m}$ light pulse intensity $E_{0}^{*}$, the observed responses of conductivity $\Delta V_{\sigma}^{*}(t), \Delta I_{\Sigma}^{*}(t)$ and diffusion current $i_{\mathrm{D}}^{*}(t)$ obey the relations below, illustrating the essential role of the two time constants $\tau_{1}^{*}, \tau_{2}^{*}$ :

$$
\begin{aligned}
& \Delta V_{\sigma}^{*}(t)=\Delta V_{\sigma 1}^{*} \exp \left(-, t / \tau_{1}^{*}\right)+ \\
& +\Delta V_{\sigma 2}^{*} \exp \left(-t / \tau_{2}^{*}\right) \\
& \Delta I_{\Sigma}^{*}(t)=\Delta I_{\Sigma \max }^{*} \exp \left(-t / \tau_{1}^{*}\right) \text { for } t<t_{\Sigma 2} \\
& \text { and }=\Delta I_{\Sigma 2}^{*} \exp \left(-t / \tau_{2}^{*}\right) \text { for } t \geqslant t_{\Sigma 2} \\
& i_{\mathrm{D} \text { front }}^{*}(t)=i_{\mathrm{D} 1 \text { front }}^{*} \exp \left(-t / \tau_{1}^{*}\right)+ \\
& +i_{\mathbf{D} 2 \text { f ront }}^{*} \sqrt{\frac{1}{t}} \exp \left(-t / \tau_{2}^{*}\right)
\end{aligned}
$$

where the slowest component $\tau_{2}^{*} \gg \tau_{1}^{*}$ is also the smallest component in all the above responses. This slow time constant $\tau_{2}^{*}$ also appears in the diffusion current responses observed under a $0.69 \mu \mathrm{m}$ back illumination of the sample; it is then preceded by a fast component whose time constant $\tau_{3}^{*}$ is somewhat greater or less than $\tau_{1}^{*}$ depending on the range of $E_{0}^{*}$. As for the photoresponses $\Delta V_{\sigma}^{*}(t)$ and $\Delta I_{\Sigma}^{*}(t)$, it should be noted that they both exhibit the fast component $\tau_{1}^{*}$; the relative contribution of this however remains highest in the constant voltage responses $\Delta I_{\Sigma}^{*}(t)$. Consistent with the analogy noted above in paragraph 3.1 on the enhanced surface contribution appearing in the photoconductance and open circuit voltage responses compared with the photoconductivity $\Delta V_{\sigma}(t)$ and photocurrent $i_{\mathrm{D}}(t)$ responses, this enhanced surface contribution may explain why a slow component $\tau_{2}^{*}$ appears rarely in the $v_{\mathrm{co}}^{*}(t)$ signals observed and obeying the relations :

$$
\begin{aligned}
v_{\text {cofront }}^{*}(t)=v_{\text {co } 1 \text { front }}^{*} \exp \left(-t / \tau_{0}^{*}\right) & + \\
& +v_{\mathrm{co} 2 \text { f ront }}^{*} \exp \left(-t / \tau_{1}^{*}\right) \\
v_{\text {co back }}^{*}(t)=v_{\text {co back }}^{*} \exp -\left(t / \tau_{3}^{*}\right) & + \\
& +v_{\mathrm{co} 2 \text { back }}^{*} \exp \left(-t / \tau_{\mathrm{c} 2}^{*}\right)
\end{aligned}
$$

where $\tau_{0}^{*} \leqslant \tau_{1}^{*}$ and $\tau_{\mathrm{c} 2}^{*}=\tau_{2}^{*}$ in the lowest range of $E_{0}^{*}$. The values, and/or variations with the $0.69 \mu \mathrm{m}$. light intensity $E_{0}^{*}$, both of the decay time $\tau_{2}^{*}$ and amplitude $\Delta V_{\sigma 2}^{*}, \Delta I_{\Sigma 2}^{*}$ of the slowest component of the photoconductivity responses correspond to the values and/or variations with $\Delta$ of the decay constant $\tau_{\sigma, \mathrm{v}(\mathrm{i}), \mathrm{c}}=\tau_{\mathrm{v}}$ and amplitudes of the sample photoresponses to the $1.06 \mu \mathrm{m}$ pulses, especially in the lowest $E_{0}^{*}$ range. With bulk carrier injection by the excited carrier diffusion in the sample, with the exception of the open circuit voltage measurements, the above results thus show that strongly absorbed light pulses also permit bulk carrier lifetime measurements in homogeneous samples when using the transient photoresponse decay methods, especially in the low level range of the induced carrier. In such cases, however, care must be taken when choosing the portion of the decay signal to be used. The effective excess carrier level $\Delta^{*}$ to which the measured lifetime refers is also difficult to estimate. However, a simple evaluation of $\Delta^{*}$ is given by the low component $\Delta V_{\sigma 2}^{*}$ amplitude of the photoconductivity responses, with $\Delta^{*}=\Delta V_{\sigma 2}^{*} / V_{\mathrm{e}}$ if $\Delta V_{\sigma 2}^{*} \ll V_{\mathrm{e}}$.

Of course, if the sample studied is a $\mathrm{n}^{+} \mathrm{p}$ structure such as a solar cell (cf. Fig. 1b), only excess carriers can be induced in the front $\mathrm{N}^{+}$region when the $W_{\mathrm{N}^{+}}$region thickness is higher than the effective light absorption length $d=1 / \alpha$. In these samples, the $i_{\mathrm{D}}(t)$ responses to $1.06 \mu \mathrm{m}$ and $0.53 \mu \mathrm{m}(T=2 \mathrm{~ns}$, $\alpha \simeq 10^{4} \mathrm{~cm}^{-1}$ ) light, as given by expressions (14) or (15), may be used in carrier lifetime measurements of the $P$ region substrate and $\mathrm{N}^{+}$emitter, respectively $[1,6]$. The increase and decay of the $v_{\text {co }}(t)$ response 
to $1.06 \mu \mathrm{m}$ and/or $0.53 \mu \mathrm{m}$ light usually leads to less precise results in such measurements [1].

The results described above deal with thick samples and silicon materials where $1.06 \mu \mathrm{m}$ light carrier excitation is produced by a one-photon band-to-band process. The response of thin silicon samples $(W=50$ $70 \mu \mathrm{m})$ to $1.06 \mu \mathrm{m}$ pulses are illustrated in ref. [4] : the measured conductivity and diffusion current photoresponses are then described by the expressions (7), (8) and (14) respectively, and also agree with the overall analysis of section 2. The photoresponses to $1.06 \mu \mathrm{m}$ light of gallium arsenide samples involve a two-photon band-to-band excitation process $\Delta \propto E_{0}^{2}$. Moreover, in these samples, one-photon impurity ionisation such as $\Delta_{\mathrm{i}} \propto E_{0}$ can appear as a competitive carrier excitation process in the lowest $E_{0}$ range. The induced light absorption coefficient and $\Delta\left(E_{0}\right)$ variations have then to be taken into account when applying the transient photoresponses to carrier lifetime $\tau(\Delta)$ studies [15].

4. Conclusion. - The analysis of the conditions under which the expressions for transient electrical photoresponses, for low (and intermediate) carrier injection and for a uniform carrier excitation in a homogeneous sample, may be reduced to simpler forms makes it possible to determine the conditions under which these photoresponses provide carrier lifetime measurements. The study then points out that the conditions of validity of the associated measurement methods clearly involve (i) the characteristics of the sample studied, (ii) the excess carrier density $\Delta$ and (iii) light pulse duration $T$ used, as well as (iv) the times $t$ considered in the response. In particular, the analysis also gives the conditions when the measured lifetimes $\tau$, which may vary with the photoresponse because of the sample boundary conditions involved, is the bulk carrier lifetime $\tau_{v}$. It also helps in determining the advantages of each method in order to find carrier lifetime in a given sample - e.g. sensitivity, validity or not above the weak carrier injection condition, sample contact contribution or not in the response. The experimental results obtained agree with the above analysis.

Moreover, the study points out some practical aspects which are to be taken into account in the choice and/or use of these methods. These aspects deal particularly with the :

(i) components involved in the experimental response, especially the fast component due to the contribution of the sample near-surface region. The latter mainly appears in the constant-voltage photoconductivity $\Delta I_{\Sigma}(t)$ and open-circuit photovoltage responses ;

(ii) interest of back illumination when using diffusion-photocurrent or open-circuit photovoltage decay methods (cf. § 3.2);

and (iii) possibility of using either strongly absorbed light (i.e. carrier injection involving the diffusion in the sample of the excited carriers) or light of subbandgap photon energy (i.e. carrier injection by interband multiphoton excitation process) in bulk carrier lifetime measurements.

From this point of view, the results also illustrate the ease that an experimental set up using the laser pulse excitation technique gives. The following remarks may aid in choosing a measurement method :

$a$ ) in the lowest excitation range, the photocurrent decay or photovoltage (especially decay) methods are easier to apply for low resistivity samples;

b) but photovoltage is to be used carefully to obtain the carrier lifetime in a given sample neutral region, and only for measurements under weak carrier injection conditions;

c) the constant-voltage photoconductivity $\Delta I_{\Sigma}(t)$ may be advantageous in the case of high impedance samples (cf. Fig. 1a);

d) the decay of the constant current photoconductivity response $\Delta V_{\sigma}(t)$ remains a valid method for studying thin samples;

e) at low excess carrier injection, the amplitude of the constant-current photoconductivity response $\Delta V_{\sigma}(t)$ may be used to calibrate the injected carrier density, especially when carrier excitation occurs near the sample surface (use of $\Delta V_{\sigma 2}$ for strongly absorbed light) or is created in the sample bulk by multiphoton excitation processes (use of $\Delta V_{\sigma \max }$ ).

\section{References}

[1] Gasset, G., Benzohra, M., Johan, A., Bielle-Daspet, D., Rev. Phys. Appl. 14 (1979) 209.

[2] Bielle-Daspet, D., Johan, A., Espioussas, F. and Gasset, G., Int. Conf. "Recombination in Semiconductors ", Solid State Electron. 21 (1978) 1618.

[3] McKelvey, J. P. and Longini, R. L., J. Appl. Phys. 25 no 5 (1954).

[4] Bielle-Daspet, D., Gasset, G. and Johan, A., Proc. of the " Photovoltaic Solar Energy Conference " (Luxembourg, sept. 1977) D. Reidel Publ. Co, p. 920-29 (1977).

[5] KuIKEN, H. K. Solid State Electron. 19 (1976) 437.

[6] Bielle-Daspet, D. and Gasset, G., Solid State Electron. 21 (1978) 1219.

[7] Lindhoim, F. A. and Tang Sah, C., J. Appl. Phys. $47 \mathrm{n}^{\circ} 9$ (1976) 4203.

[8] Dhariwal, S. R., Kothari, L. S. and Jain, S. C., Solid State Electron. 20 (1977) 297.
[9] Bielle-Daspet, D., Benzohra, M. and Gervais de Lafond, Y., Solid State Electron 21 (1978) 909.

[10] Bielle-Daspet, D., Espioussas, F., Johan, A., Coll. Soc Française de Phys., Toulouse, juin 1979.

[11] Wertheim, G. K., Phys. Rev. 109 (1958) 1086.

[12] Baiker, J. A., Phys. Rev. 129 no 3 (1963) 1174.

[13] Parrot, J. E., IEEE Trans. Electron Devices, ED 21 (1974) 89

[14] The theoretical decrease $\mu_{\mathrm{p}}(\Delta)$ is calculated using the phenomenological expression given for ohmic mobility as function of impurity concentration, in : GWYN, C. W., SChARFETTER, D. L., WirTh, J. L., IEEE Trans. Nucl. Sci. NS 14 (1967) 153.

[15] Espioussas, F., thèse Doct. Ing., Toulouse, sept. 1979, Mesures des propriétés électriques du silicium et de l'arséniure de gallium à partir des photoréponses électriques transitoires à une excitation par laser pulsé. 